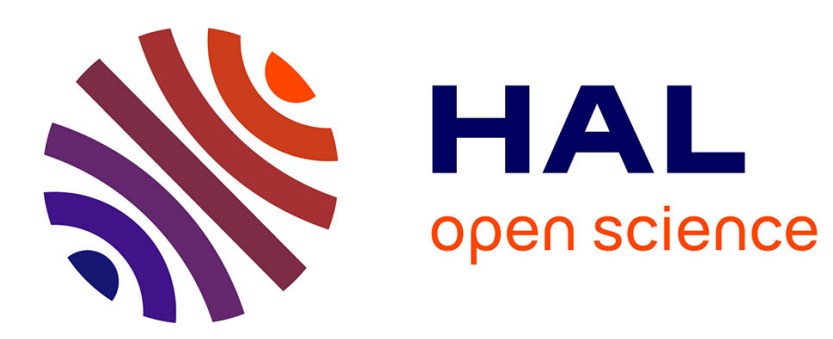

\title{
A Low Power Ku Phase Locked Oscillator in Low Cost 130 nm CMOS Technology
}

\author{
Alessandro Magnani, Mattia Borgarino, Christophe Viallon, Thierry Parra, \\ Gilles Jacquemod
}

\section{> To cite this version:}

Alessandro Magnani, Mattia Borgarino, Christophe Viallon, Thierry Parra, Gilles Jacquemod. A Low Power $\mathrm{Ku}$ Phase Locked Oscillator in Low Cost $130 \mathrm{~nm}$ CMOS Technology. Microelectronics Journal, 2014, 45 (6), pp.619-626. hal-01005978

\section{HAL Id: hal-01005978 https://hal.science/hal-01005978}

Submitted on 13 Jul 2014

HAL is a multi-disciplinary open access archive for the deposit and dissemination of scientific research documents, whether they are published or not. The documents may come from teaching and research institutions in France or abroad, or from public or private research centers.
L'archive ouverte pluridisciplinaire HAL, est destinée au dépôt et à la diffusion de documents scientifiques de niveau recherche, publiés ou non, émanant des établissements d'enseignement et de recherche français ou étrangers, des laboratoires publics ou privés. 


\title{
A Low Power Ku Phase Locked Oscillator in Low Cost $130 \mathrm{~nm}$ CMOS Technology
}

\author{
Alessandro Magnani ${ }^{\mathrm{a}, \mathrm{b}}$, Mattia Borgarino ${ }^{\mathrm{a},}$, Christophe Viallon ${ }^{\mathrm{b},}$, Thierry \\ Parra $^{\mathrm{b},}$, Gilles Jacquemod ${ }^{\mathrm{c}}$ \\ ${ }^{a}$ University of Modena and Reggio Emilia (Modena, Italy) \\ ${ }^{b}$ University of Toulouse; UPS; LAAS (Toulouse, France) \\ ${ }^{c}$ University of Nice Sophia Antipolis (Valbonne, France)
}

\begin{abstract}
This paper reports on a Ku low-power integer-N phase locked oscillator designed to investigate the potentialities of a low-cost $130 \mathrm{~nm}$ CMOS technology for video broadcasting and radiometry applications. The design and the characterization of the prototype are described and the main performances are reported and compared to literature. The PLO generates an output tone in the $14.2 \mathrm{GHz}$ to $15.1 \mathrm{GHz}$ frequency range. The phase noise is $-68.9 \mathrm{dBc} / \mathrm{Hz}$ for an offset frequency of $100 \mathrm{kHz}$ from a $15 \mathrm{GHz}$ carrier, and can be enhanced of about $20 \mathrm{~dB}$. The circuit core sinks $23.7 \mathrm{~mA}$ from $1.2 \mathrm{~V}$ supply.
\end{abstract}

Keywords: $\mathrm{Ku}$ band, CMOS, DVBS, PLO, microwave radiometer

\section{Introduction}

Several mass market applications, as for example, Standard and High Definition TeleVision (SDTV and HDTV), interactive multimedia, data content distribution and professional TV applications use a portion of $\mathrm{Ku}$ band spectrum reserved for point to point and broadcasting satellite communications. Nowadays, in satellite receivers, the signal is picked up by the dish antenna and then amplified and down-converted by a low noise front-end. This block is frequently built using discrete compound semiconductor High Electron Mobility Transistors (HEMT) and Dielectric Resonator Oscillators (DRO), as well. This discrete approach is expensive due to components, assembling operations, and the resonator manual tuning [1]. A silicon-based monolithic integrated receiver offers advantages in term of cost and size, specially when an integrated oscillator is used to replace the DRO. Nevertheless, 
performance limitations of integrated solutions are still restricting this kind of solution for penetrating high frequency mass market applications such as Digital Video Broadcasting Satellite (DVB-S) [2]. Some efforts have led to several prototypes as a monolithic $0.8 \mu \mathrm{m}$ bipolar technology Low Noise Block (LNB) by STMicroelectronics [1], and more recently, a 0.25 $\mu \mathrm{m}$ SiGe:C commercially available BiCMOS technology LNB reported by NXP Semiconductors [2]. Avoiding the use of bipolar transistors is of great interest as well, which is the aim of [3] and [4], where a $0.18 \mu \mathrm{m}$ CMOS LNA/down-conversion mixer chain and a LNB, respectively are claimes. In all reported examples, the frequency synthesizer used to generate the local oscillator (LO) signal is a Phase Locked Loop (PLL). This kind of implementation requires a programmable frequency divider in the feedback loop. To easier demonstrate the potentiality of a technology for a given application where a local clock and/or a local frequency are required, the use of a Phase Locked Oscillator $(\mathrm{PLO})$ is a good alternative because the frequency divider in a PLO exhibits a fixed modulus, making in this way easier its design. The present paper reports on the design and the characterization of a Ku-band PLO realized with a bulk $130 \mathrm{~nm}$ CMOS technology. The operation frequency was chosen so that to test its potentiality for both up-link $(12.9 \div 18.4 \mathrm{GHz})$ and down-link $(10.7 \div 12.75 \mathrm{GHz})$ frequency band. Particular attention has been paid on the phase noise which is the hardest specification to be satisfied. The modulations scheme (APSK) adopted in DVB-S standard exhibits indeed a round constellation where excessive rotational errors due to excessive phase noise would produce a burst of error [5]. The PLO can be also used as local oscillator in the architecture of a microwave radiometric front-end [6]. Several applications in the microwave radiometry field, such as industrial harsh plants, where conventional sensors can not be employed, or automotive safety, require cost and size reductions of the radiometer. For example, in order to keep low the cost, the emitted black-body radiation is usually detected with a low cost printed antenna array $[6,7]$, which size depends on the operation frequency. A PLO working at higher frequency allows therefore a reduction in the antenna size. For a $8 \times 8$ array patch-antenna with a gain of $25 \mathrm{~dB}$, when the operation frequency moves from X-band to Ku-band, as in the present case, the antenna area shrinks of 2.5 times, from $10 \times 10$ inch $^{2}$ to $6.3 \times 6.3$ inch $^{2}$ [8]. Microwave radiometry is considered also an interesting solution for wild fire detection. In order to minimize the maintenance cost of the batteries, the microwave radiometer collocated in each node of the smart sensor network distributed on the wild area should be energy independent 
as much as possible, exploiting renewable energy sources, as integrated microphotovoltaic cells or harvesting circuits [9]. Dissipated power reduction is therefore a further parameter of paramount importance. Using, as in this case, low power technology allows to solve this constraint, making easier to target the energy independence of each node of the smart sensor network. When bias voltage moves from a $3.3 \mathrm{~V}$ of a $0.35 \mu \mathrm{m}$ technology to a $1.2 \mathrm{~V}$ of a $130 \mathrm{~nm}$ technology, a significant reduction of the dissipated power has to be expected. Therefore, the use of a higher operation frequency and more scaled technology with respect to those reported in [6] allows to address the above discussed advantages of reduction in size and dissipated power. The present paper is organized as follows. Section 2 gives brief description of the used technology. The PLO architecture and the design of each building block are described in Section 3 and 4, respectively. Experimental results are summarized in Section 5 followed by some considerations in Section 6 . Finally conclusions are drawn in Section 7 .

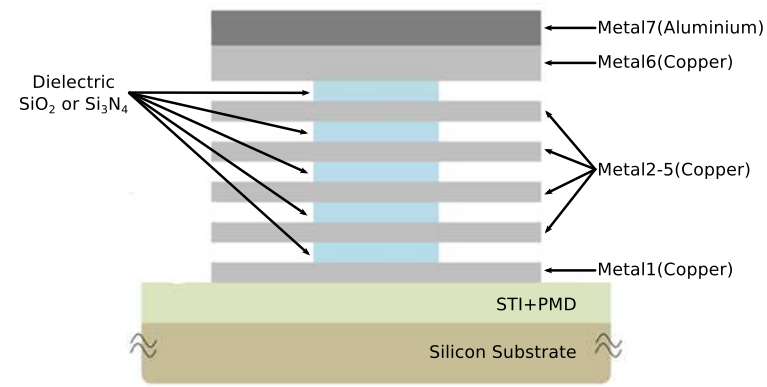

Figure 1: $130 \mathrm{~nm}$ CMOS STM technology (HCMOS9GP).

\section{IC Technology}

The circuit is designed to fit with the commercial HCMOS9GP technology from STMicroelectronics. It is a triple well bulk CMOS technology with multiple voltage threshold transistors, for low leakage or high speed purposes, featuring an effective gate length of $130 \mathrm{~nm}$. As shown in Figure 1, the back-end features six copper layers with low-k inter-level dielectric and one aluminium layer on top [10], [11], [12]. The bias supply is typically $1.2 \mathrm{~V}$ even if the $2.5 \mathrm{~V}$ option is also available [11]. For the present design, low leakage, low threshold voltage transistors have been used with a supply voltage of $1.2 \mathrm{~V}$. Several kinds of resistors are also available, as diffusion, salicied or 
unsalicied poly, and high resistivity poly resistors [13]. MIM capacitors, spiral inductors, and varactors are also available.

\section{PLO Architecture}

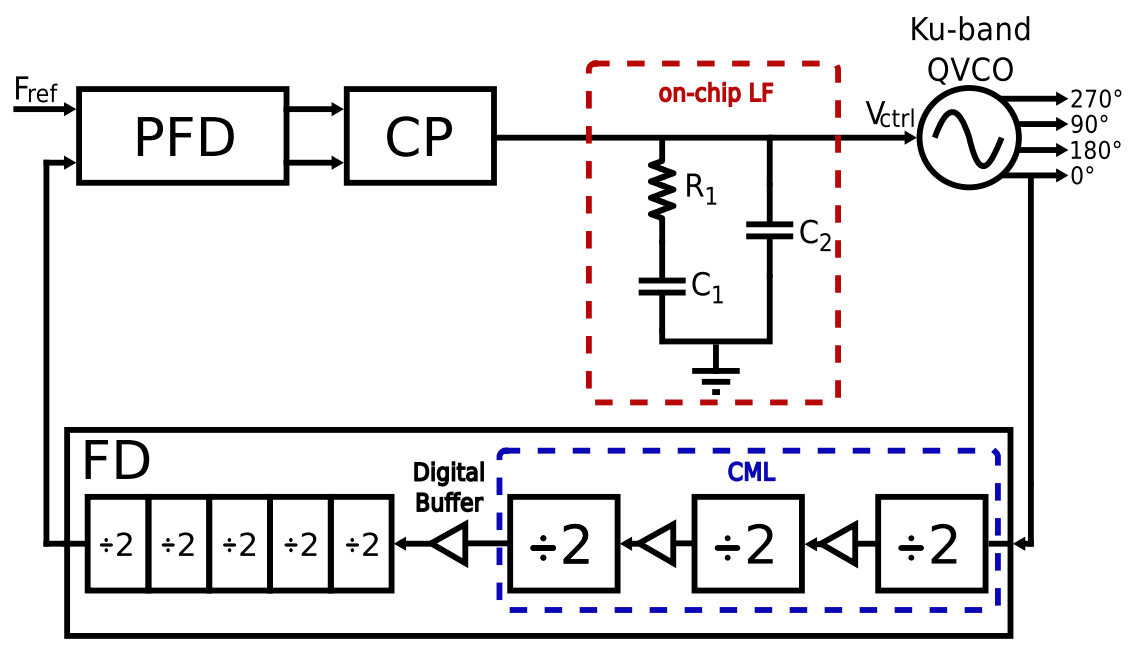

Figure 2: Phase Locked Oscillator Blocks Diagram.

Figure 2 depicts the building block diagram of the designed PLO. It is constituted by a Phase Frequency Detector (PFD), a Charge Pump (CP), a second order Loop Filter (LF), a Frequency Divider (FD), and a Quadrature Voltage Controlled Oscillator (QVCO). The difference with respect to an $\mathrm{N}$-integer PLL is that the FD in the PLO exhibits a fixed modulus while in the PLL the FD is constituted by two blocks: the prescaler, a dualmodulus frequency divider guaranteeing a frequency resolution equal to the reference frequency, and a programmable section, usually implemented with down counters. It is worth here reminding that the design of the $\mathrm{VCO} / \mathrm{FD}$ interface is the challenging step in the design flow of the loop, specially when the first block of the FD is the pre-scaler. The PLO architecture with its fixed modulus FD offers therefore the advantage of making a bit less critical the design of this interface. Once the potentiality of the addressed technology for a given application is demonstrated, efforts can be spent to design a programmable FD, to obtain a PLL from the PLO, in the case a PLL would be required.The frequency division ratio (256) of the FD is set for enabling the generation of a Ku-band tone from an external reference frequency $f_{\text {ref }}$ 
of about $60 \mathrm{MHz}$. The frequency divider is implemented as eight division-bytwo stages. The first three stages are designed using a Current Mode Logic (CML) to achieve high operation frequency. Each CML stage is buffered in order to be able to drive the following divider stage. The division chain is completed with five low power digital frequency dividers realized with transmission gate registers. Because of the QVCO implementation, four output phases are available at PLO outputs. Finally, it is worth noticing that the loop filter is integrated on die for low cost considerations.

\section{PLO Design}

\subsection{Quadrature $V C O$}

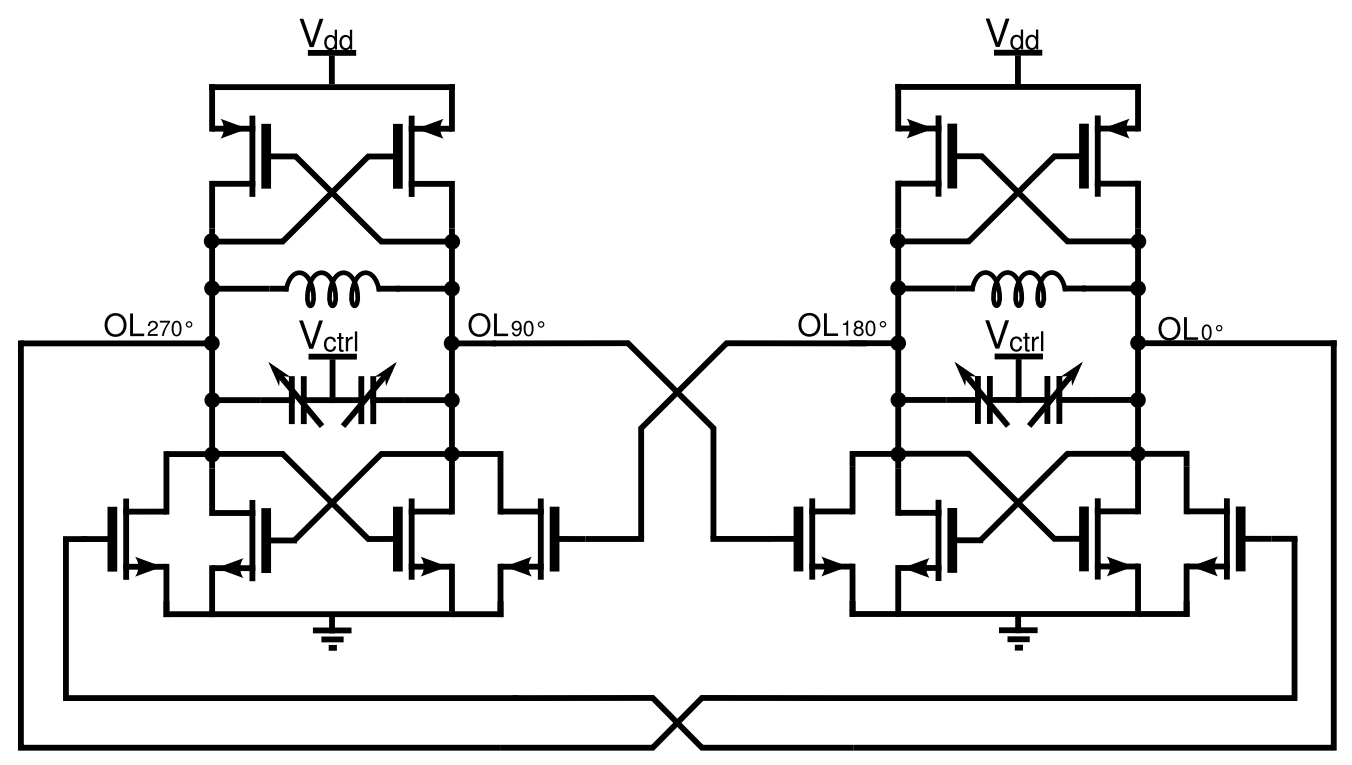

Figure 3: Schematic of the Quadrature VCO.

For generating signals in quadrature, the main techniques usually used are poly-phase filters, ring oscillators or frequency dividers. Nevertheless, in the present work two cross-connected symmetric LC VCOs has been preferred, because of their good phase noise performances.

The schematic of the QVCO is depicted in Figure 3 and was previously reported in [14]. Here some features and performances are quickly reminded; more details are available in [14]. The circuit is biased without current mirror to minimize the phase noise, the power consumption, and also to avoid any 
automatic control circuit. All transistors exhibit the minimum gate length. The width of PMOS transistors must be wide enough to ensure robustness against fabrication tolerances. The octagonal inductor in the tank exhibits an inductance of about $290 \mathrm{pH}$ and a maximum quality factor of 27 . The tuning capability is achieved using two 20 fingers MOS varactors with a minimum gate length of $350 \mathrm{~nm}$. For a carrier frequency $\left(f_{\text {carrier }}\right)$ of $15 \mathrm{GHz}$, the dissipated power $\left(P_{D C}\right)$ is $11 \mathrm{~mW}$ and the measured phase noise $\mathrm{L}(\Delta f)$ is $-106 \mathrm{dBc} / \mathrm{Hz}$ for a carrier frequency offset $(\Delta f)$ of $1 \mathrm{MHz}$.

\subsection{Frequency Divider}

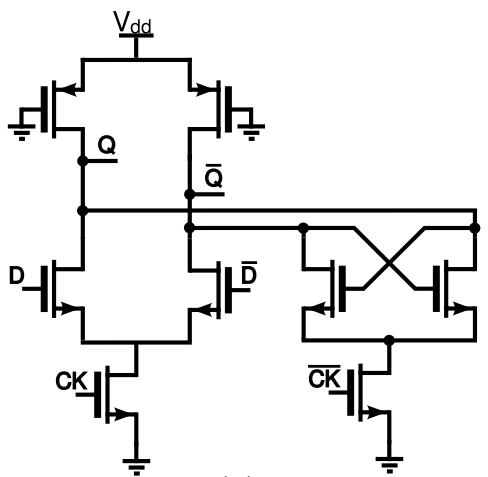

(a)

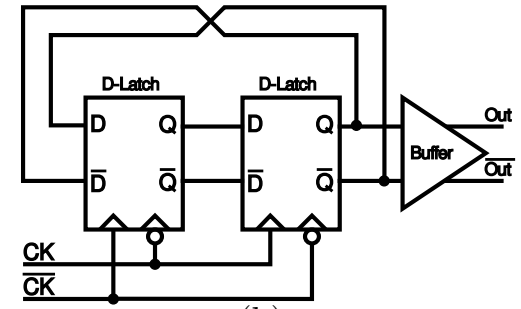

(b)

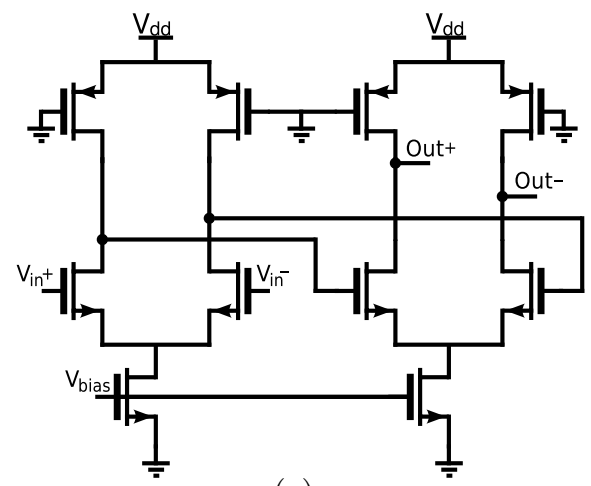

(c)

Figure 4: Schematic of: (a) CML latch, (b) Master-Slave divide-by-two frequency divider, (c) CML buffer

As previously stated, the frequency divider is composed of eight division stages. Because it operates at high frequencies, each of the first three divisionby-two stages is implemented through the CML latch depicted in Figure 4(a). 
The frequency division is carried out by two latches arranged in a masterslave configuration and closed in feedback, as depicted in Figure 4(b). The schematic of the output buffer required to drive the following division stage is shown in Figure 4(c) [15]. It is worth noticing here that the use of CML structures is well suited since their differential intrinsic topology contributes to reject the common mode noise injected from the supply and the substrate. Once translated in PLO performances, this rejection provides lower jitter and therefore lower phase noise [16]. In the present work the CML latch, and of course the resulting frequency division CML chain, is inductor-less, using PMOS transistors as loads to minimize the layout area of circuits. Because of the low voltage bias, the traditional tail current bias is removed [17]. The inductor-less solution sounds reasonable, since it can provide a $130 \mathrm{~nm}$ CMOS static frequency divider operating up to $45 \mathrm{GHz}$ frequency. Inductors become mandatory when a $130 \mathrm{~nm}$ CMOS frequency divider should target millimeter wave frequencies [18]. Another design issue of paramount importance is the correct extraction and the minimization of parasitics at the interface between the VCO and the frequency divider. Unpredicted and/or false parasitics can translate into a frequency mismatch between the tuning range of the VCO and the sensitivity range of the frequency divider, making impossible the lock of the PLO. Therefore, a compact front-end of the frequency divider allows the optimization of this interface by reducing parasitics from the layout of this very critical interface. Because of this criticality the CML latch must be very carefully laid out. In order to assess the robustness of the circuit against technology dispersions, several post-layout simulations have been carried out under RC parasitic worst case of the VCO/FD interface and for several corner cases of transistor transconductance values. Figure 5 plots simulated output frequency versus input frequency of the first CML divider stage. A slope of 0.5 certifies the proper operation of this divide-by-two stage. Figure 5 shows that in the $6 \mathrm{GHz}$ to $16 \mathrm{GHz}$ frequency range, the CML divider stage is able to correctly work not only under the typical corner case (TT) but also under the Slow-Slow (SSA) and the Fast-Fast (FFA) corner cases, i.e. when the transistor transconductance is lower and higher than its typical value, respectively. Note that the frequency range where the circuit correctly works is larger (narrower) than the typical case when the transistors are fast (slow). The SSA case is therefore the most critical one. Similar simulations have been carried out for the other CML stages.

As far as the implementation of the last five division-by-two stages operating at lower frequency, dynamic CMOS latches are used as base cells where 


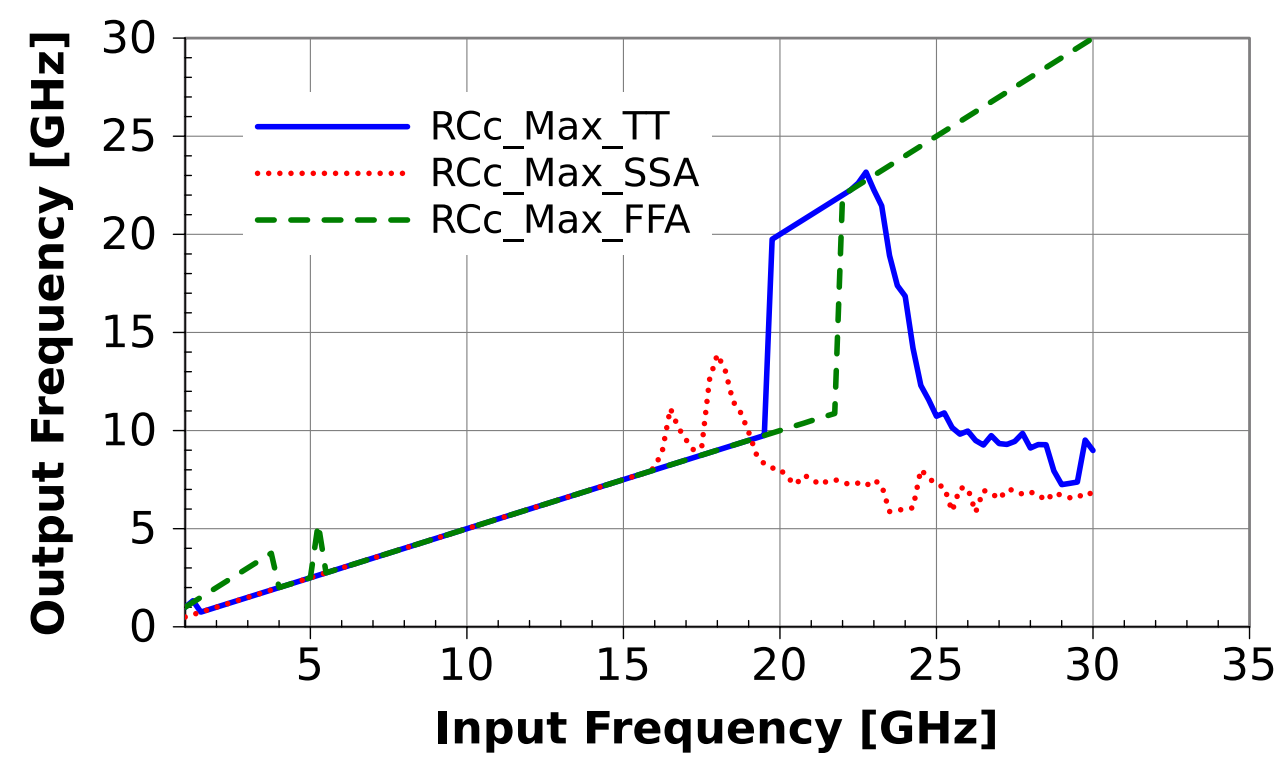

Figure 5: Output frequency versus input frequency of the first CML divide-by-two frequency divider for several corner cases under worst case parasitic extraction.

the transmission gates sample the input data and synchronize the transparent and opaque states. As depicted in Figure 2, a digital buffer, simply composed by the cascade of two inverters, interfaces the CML and the low frequency sections. The whole division chain exhibits a simulated power consumption of about $10.5 \mathrm{~mW}$. The small size of the whole frequency divider (Figure 6) of $44 \times 154 \mu^{2}$ is mainly due to the inductor-less approach. Because in these conditions the connection between the QVCO output and the frequency divider can be very short (only few microns as pointed out in Figure 6), RLC parasitic components of the connection are minimized ensuring a good frequency matching between FD and QVCO.

\subsection{Phase Frequency Detector, Charge Pump and Loop Filter}

Figure 7(a) shows the schematic of the designed Falling-Edge (FE) PFD [19]. Thanks to the absence of a reset signal, as more traditional PFDs often requires, this configuration is dead zone free. This property leads to a better phase noise of the PLO. It is worth noticing that the True Single Phase Clock (TSPC) solution is dead zone free as well, but its implementation requires more transistors than the FE solution [19]. As it combines good phase noise and compact layout, the FE solution has been adopted in the 


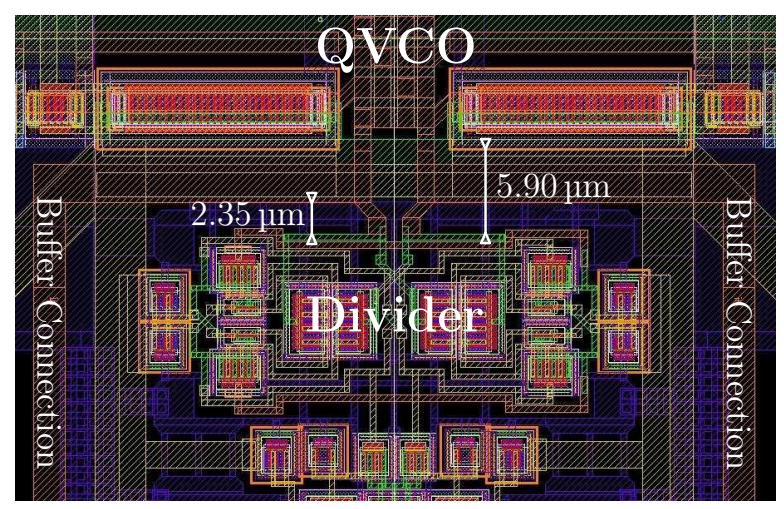

Figure 6: Detailed layout of the interface between the QVCO and the frequency divider.

present work. Further advantages offered by this structure are low dissipated power and high speed operation. It is worth pointing out that to avoid dead zone and get a good linearity of the phase characteristic, the FE PFD should compare signals exhibiting the same duty cycle with a value in the range of $50 \%$ [19], which is the case of the PLO reported in the present paper. Under these conditions, the simulated phase noise of the designed FE PFD is about $-173.8 \mathrm{dBc} / \mathrm{Hz}$ at frequency offset of $1 \mathrm{MHz}$, outperforming the $-168.8 \mathrm{dBc} / \mathrm{Hz}$ claimed in [19]. Moreover, it has to be pointed out that the PFD schematic depicted in Figure 7(a) exhibits a differential structure. This topology not only makes the PFD robust against common mode noise sources but also provides up and down signals in both true and false forms, so that a differential CP can be driven.

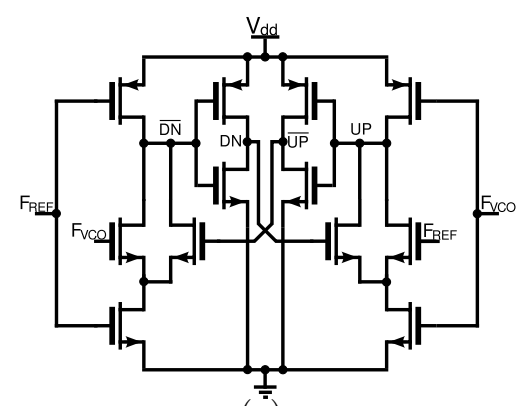

$(\overline{\bar{a}})$

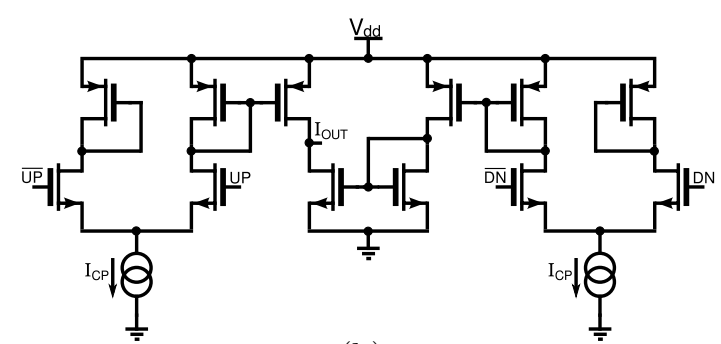

(b)

Figure 7: Schematic of the designed PFD (a) and charge pump (b).

A differential topology is selected for the CP, which is able to improve 
the common mode noise rejection [16]. The schematic of the designed CP is shown in Figure 7(b). Longer channel transistors are used in the current mirror to improve the current matching, as much as possible [20]. Finally, as known, the values of capacitance and resistance implemented in the loop filter (see Figure 2) depend on the desired open loop gain. More precisely, the capacitances are directly proportional to $K_{\varphi} \cdot K_{V C O} /\left(\omega_{B W}\right)^{2} \cdot N$, where $K \varphi$ is the gain of the CP, $\omega_{B W}$ the bandwidth of the PLO, and $\mathrm{N}$ is the division ratio of the frequency divider. Considering $K_{\varphi}=I_{C P} / 2 \pi$ where $I_{C P}$ is the $\mathrm{CP}$ current, values of the capacitances are also directly proportional to this current. Hence, the size of the loop filter is kept small by setting $I_{C P}$ to $25 \mu \mathrm{A}$. This low current value allows a second order loop filter to be integrated on chip $\left(C_{1}=44 \mathrm{pF}, R_{1}=27.09 \mathrm{k} \Omega\right.$ and $\left.C_{2}=3.39 \mathrm{pF}\right)$. The resulting bandwidth and phase margin are $500 \mathrm{kHz}$ and 60 degrees, respectively. To shrink the filter size as much as possible, polysilicon n-well capacitors are used for their higher capacitance-area ratio.

\section{Experimental Result}

Figure 8(a) shows the microphotograph of the fabricated prototype where each building block of the PLO is highlighted. Note in higher chip side the buffers driving the $50 \Omega$ load of the external instrumentation.The GSGSG pads for the differential $\mathrm{RF}$ output $\left(\mathrm{OL}_{90^{\circ}}, \mathrm{OL}_{270^{\circ}}\right)$ are also visible on the top of the chip. To keep the circuit as symmetric as possible for the best balanced operation of the QVCO, two dummy buffers are connected on both other RF outputs. These outputs are not available on pads for experimental test simplification. Both pads on the left are implemented for the measurement of the frequency divider output signals $\left(\operatorname{Div}_{Q}\right.$ and $\left.\operatorname{Div}_{I}\right)$. On the bottom, pads are visible for the reference signal and power supply. The size of the whole chip is $920 \times 1010 \mu^{2}$, pad enclosed. The PLO active area without buffers occupies an area of about $450 \times 900 \mathrm{\mu m}^{2}$, which is quite compact.

For sake of test simplification and measurements quality, the die is mounted on a PCB test board. The bias pads as well as the reference low frequency pad are wire-bonded to interconnection lines. High frequency output signals are measured through microwave coplanar probes. Thanks to this experimental set-up only one differential RF probe was required. The bias was correctly filtered with several microcapacitors located as close as possible to the die. The bias voltage was supplied by a TTi Thurlby Thandar Instruments PL330 32V-3A Power Supply Unit. The measurements were carried 


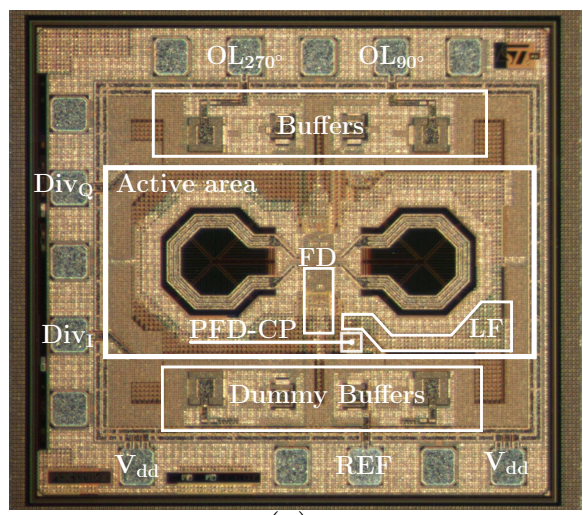

(a)

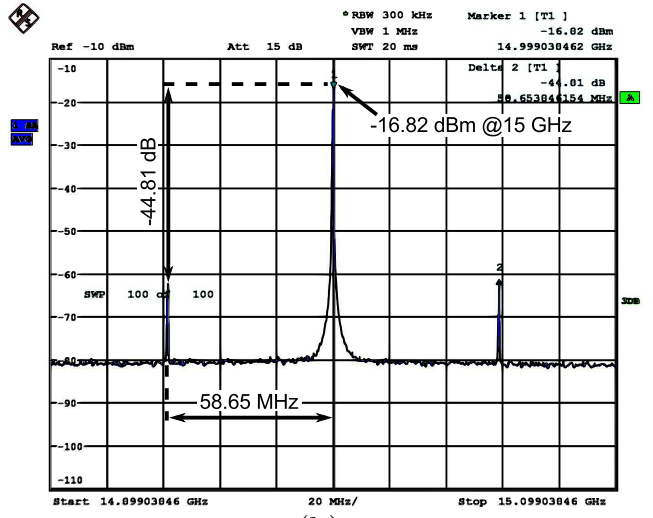

(b)

Figure 8: (a) Die microphotograph of the fabricated PLO, (b) Measured output spectrum

out inside a Faraday cage, in order to minimize possible interferences from parasitic signals. The differential output is applied to the single-ended input of the Rohde\&Schwarz FSU67 Spectrum Analyzer by picking up only one signal and closing the other one on a $50 \Omega$ load. The tuning external reference frequency is obtained from the Marconi instruments 2042 low noise signal generator $(10 \mathrm{kHz} \div 5.4 \mathrm{GHz})$. Figure $8(\mathrm{~b})$ shows the measured spectrum. By sweeping the external reference frequency, a range of $f_{\text {carrier }}$ from $14.2 \mathrm{GHz}$ to $15.1 \mathrm{GHz}$ was observed, which matches the expected tuning range. The PLO delivered a power of about $-16.8 \mathrm{dBm}$ on a $50 \Omega$ load, and it was biased with a current of $23.7 \mathrm{~mA}$ from $1.2 \mathrm{~V}$ supplied voltage. The phase noise was measured using the Agilent Technology signal source analyzer E5052B $(10 \mathrm{MHz} \div 7 \mathrm{GHz})$ associated with the microwave downconverter E5053A $(3 \mathrm{GHz} \div 26.5 \mathrm{GHz})$. Figure 9 shows the measured phase noise (red curve) for $f_{\text {carrier }}=15 \mathrm{GHz}$ in the frequency span $\Delta f$ from $10 \mathrm{~Hz}$ to $40 \mathrm{MHz}$. The figure shows that the PLO exhibited a phase noise $L(\Delta f)=$ $-68.9 \mathrm{dBc} / \mathrm{Hz} @ \Delta f=100 \mathrm{kHz}, L(\Delta f)=-86.3 \mathrm{dBc} / \mathrm{Hz} @ \Delta f=1 \mathrm{MHz}$ and $L(\Delta f)=-122.2 \mathrm{dBc} / \mathrm{Hz} @ \Delta f=10 \mathrm{MHz}$.

\section{Discussion}

Figure 9 shows a very good agreement between measured (red curve) and simulated (dotted blue curve) phase noise, indicating that the PLO correctly works. The simulated spectrum is obtained by taking into account the single contribution to the phase noise of the QVCO, the frequency divider, the 


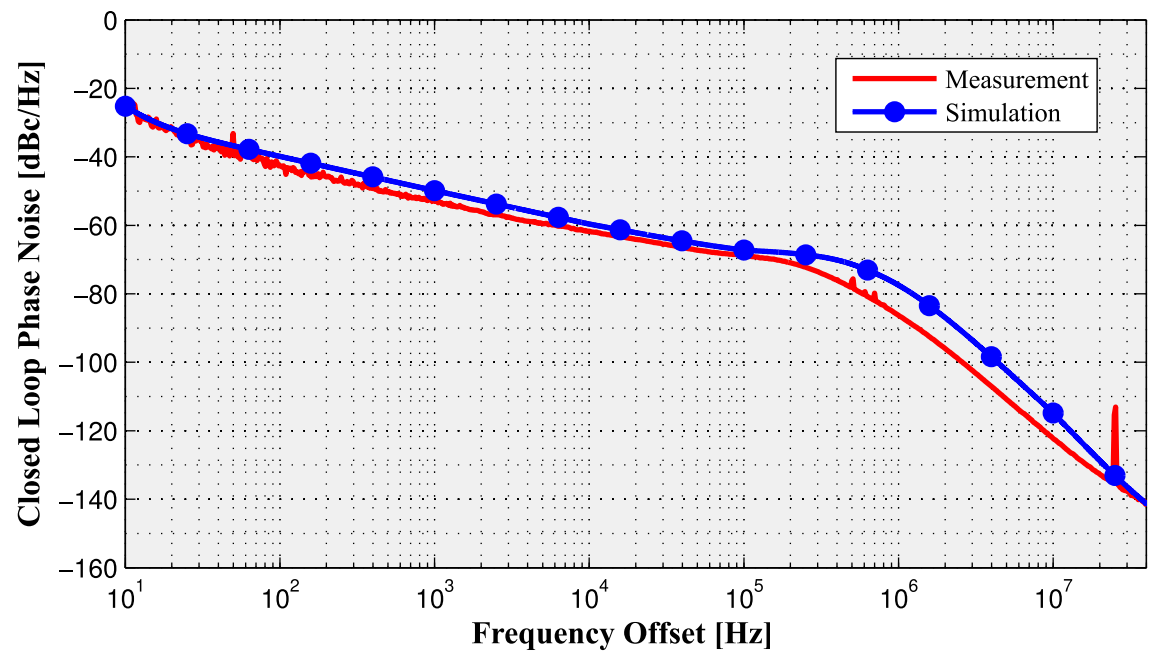

Figure 9: Comparison between measured and simulated phase noise.

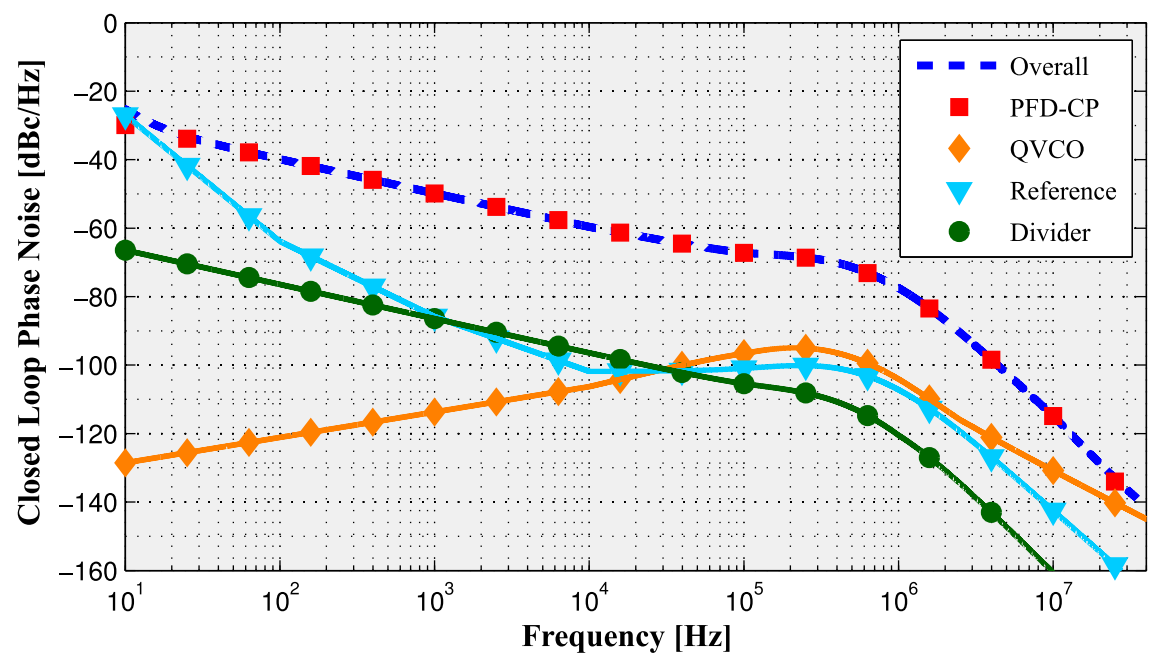

Figure 10: Simulated closed loop phase noise contributions.

PFD, and the CP. The contribution of each building block is simulated as in lock condition at transistor level and then introduced in a linear model of the PLO described using MATLAB ${ }^{\circledR}$ code and Simulink ${ }^{\circledR}$ models. With the transfer functions of this linear model and the noise contributions of each building block as an input, the developed tool computes both the open and 


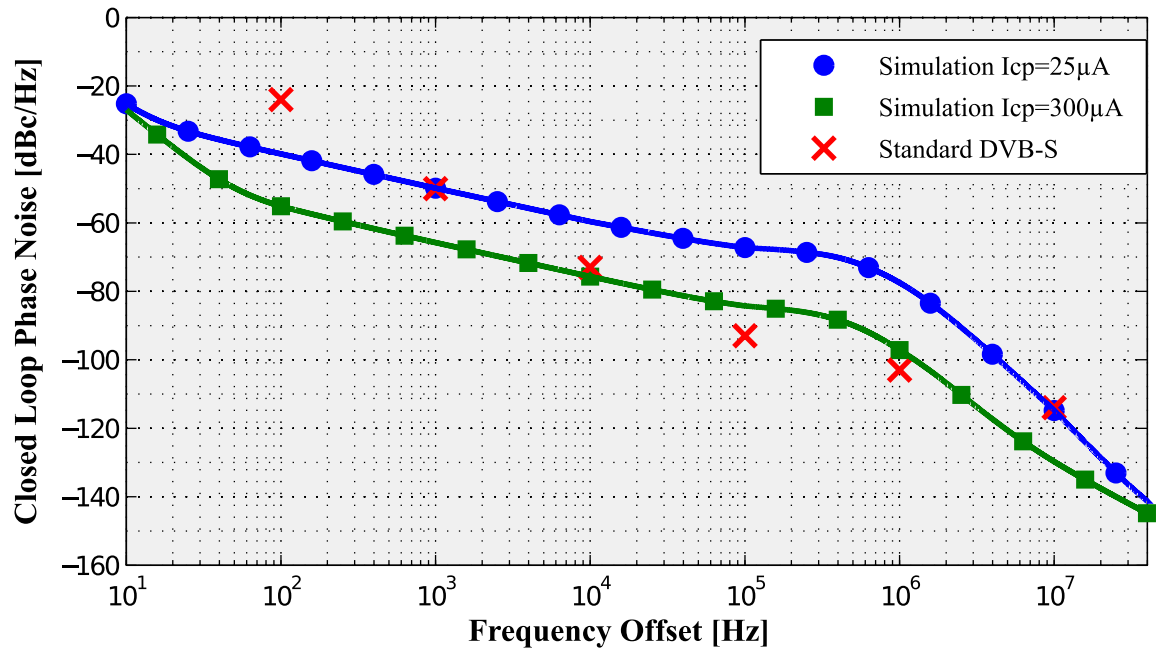

Figure 11: Comparison between the simulated phase noise with $25 \mu \mathrm{A}$ and $300 \mu \mathrm{A}$ charge pump current; red symbols are the DVB-S standard phase noise mask.

closed loop responses together with the loop design specification, the zeropole information, and the phase noise spectra as well [21]. Each simulated phase noise contribution is reported in Figure 10, where the contribution of the reference signal is reported (sky-blue curve), as well. From these results, the $\mathrm{PFD} / \mathrm{CP}$ block can be identified as the main contributor to the PLO overall phase noise.

In order to discriminate between PFD and CP, the PFD phase noise performance has been investigated using the following Figure-of-Merit for PFD $\left(\right.$ FoM $\left._{\mathrm{PFD}}\right)$ reported in [22]:

$$
\operatorname{FoM}_{\mathrm{PFD}}(d B c / H z)=L(\Delta f)-20 \log _{10} N-10 \log _{10} f_{s}
$$

where $\mathrm{N}$ is the division ratio of the frequency divider and $f_{s}$ the external reference frequency. Using this FoM $\mathrm{PFD}_{\mathrm{P}}$ to compare the performances with literature allows to find the circuit section that must to be improved; in our case this part resulted to be the CP. Then, further investigation on the CP phase noise was carried out. As indicated in [23], the phase noise contribution of the CP is referred to the PLL input by dividing the total average noise at the CP output by the gain $K_{\varphi}$ of this device. The following phase noise expression is derived in [23]: 


$$
\theta_{n}=2 \pi \sqrt{\left[\frac{2 K \mu}{L^{2} f I_{C P}}+4 k T\left(\frac{2}{3}\right) \sqrt{\left.\frac{2 \mu C_{O X} W}{I_{C P}^{3} L}\right] \cdot\left(\frac{t_{C P}}{T_{0}}\right)}\right.}
$$

where $\mathrm{K}$ is a proportionality constant depending on the process, $\mathrm{L}$ is the channel length of the $\mathrm{CP}$ transistors, $\mu$ is the channel electron mobility, and $t_{C P}$ is the interval time during which both current sources of the charge pump are on during each period $T_{0}$. Equation (2) indicates that, even if a low maximum value of $I_{C P}$ allows lower total average noise, as reported in [16], for lower phase noise a higher $I_{C P}$ value is better [23]. Equation (2) indeed shows that the phase noise is inversely proportional to $I_{C P}$. Figure 11 compares two phase noise spectra simulated for the previous $I_{C P}$ value of $25 \mu \mathrm{A}$ and for a higher value of $300 \mu \mathrm{A}$. These spectra are then compared with the DVB-S standard phase noise limits (red symbols) [5]. A phase noise reduction of about $20 \mathrm{~dB}$ is observable as a consequence of increasing $I_{C P}$, demonstrating the importance of the $\mathrm{CP}$ contribution to the overall PLO phase noise. Moreover, Figure 11 points out that the higher value $I_{C P}=300 \mu \mathrm{A}$ moves the phase noise fair close to the limits set by the DVBS standard. In particular, the PLO phase noise is better than the standard for offset frequency lower than $10 \mathrm{kHz}$, and for offset frequency higher than $1 \mathrm{MHz}$. In the $10 \mathrm{kHz} \div 1 \mathrm{MHz}$ offset frequency range, the obtained phase noise is fairly comparable with the standard. As already pointed out, since the capacitances are directly proportional to the gain of the charge pump, a trade-off between the loop filter size and the phase noise performance must be found. In particular, a $I_{C P}=300 \mu \mathrm{A}$ would require to increase by a factor of about twelve $\left(C_{1}=527.7 \mathrm{pF}, R_{1}=2.25 \mathrm{k} \Omega\right.$ and $C_{2}=40.82$ pfarad $)$ the value of the capacitances. For instance, the loop filter of the measured prototype (Figure 8(a)) occupies an area of about $15800{\mu \mathrm{m}^{2}}^{2}\left(0.016 \mathrm{~mm}^{2}\right)$; whereas for $I_{C P}=300 \mu \mathrm{A}$, the occupied area of this filter increases to about $189200 \mathrm{\mu m}^{2}\left(0.189 \mathrm{~mm}^{2}\right)$.

Even if the obtained phase noise performance have been previously compared with the DVB-S standard phase noise mask in terms of phase noise spectra, under a telecom point of view an evaluation of the noise performance in terms of integrated phase noise is also of interest, being closer related to the quality of the received constellation in the base band section. To this aim a comparison with other silicon-based PLLs already reported in the literature is carried out by using the following Figure-of-Merit [22]: 


$$
\operatorname{FoM}_{\mathrm{PLL}}(d B)=10 \log _{10}\left[\left(\frac{\sigma_{t, P L L}}{1 s}\right)^{2} \cdot\left(\frac{P_{D C}}{1 m W}\right)\right]
$$

where $\sigma_{t, P L L}$ is the RMS jitter and $P_{D C}$ is the DC power consumption. When only the phase noise spectrum is reported, a short script, developed in Matlab code, computes the jitter through the following formula [22]:

$$
\sigma_{t, P L L}^{2}=\frac{1}{2 \pi^{2} f_{\text {carrier }}^{2}} \int_{0}^{\infty} L_{P L L}(\Delta f) d \Delta f \cong \frac{1}{2 \pi^{2} f_{\text {carrier }}^{2}} \int_{W} L_{P L L}(\Delta f) d \Delta f
$$

where $L_{P L L}$ is the phase noise of the PLL and $\mathrm{W}$ is the finite offset frequency integration range. The used value of $\mathrm{W}$ for each reference is listed in Table 1. In the limits of the data available in each reference, efforts are made to keep $W$ as uniform as possible (about $10 \mathrm{kHz} \div 40 \mathrm{MHz}$ frequency range), in order to get a homogeneous comparison. Figure 12 shows the computed results where each reference is related to the DC power consuming and jitter variance. Even if the operation frequency of the circuit $(38 \mathrm{GHz})$ claimed in [24] is about two times the operation frequency addressed in the present work $(15 \mathrm{GHz})$, reference [24] has been accounted for, because of the very low values of jitter and FoM.The FoM of the proposed PLO is well located in the comparison with the literature, since it is close to $-215 \mathrm{~dB}$ in the case of a $I_{C P}=25 \mu \mathrm{A}$ and falls down to about $-230 \mathrm{~dB}$ in the case of a $I_{C P}=300 \mu \mathrm{A}$. Figure 12 shows also the jitter variance limit (red line) calculated with equation (4) using the phase noise limit of the DVBS standard, previously depicted in Figure 11. One can see that the presented PLO comes closer to the two best performances [24], [25] and to the DVBS jitter limit when an increase of the loop filter silicon area is acceptable, in order to increase $I_{C P}$.

This is a very interesting result, because the fabricated PLO prototype demonstrates that a pure bulk CMOS technology, a $130 \mathrm{~nm}$ CMOS technology in the present case, exhibits very promising potentialities for the fabrication of a PLL compliant with the DVB-S standard.

A more detailed performances comparison is summarized in Table 1. Note the low power consumption of the PLO described in the present paper when the buffer contribution is neglected; its dissipated of $28 \mathrm{~mW}$ is challenged only by the PLL reported in [25] and several times lower, between two and sixteen times, the power dissipated by the other PLLs. This result in addition with the small occupied silicon area give to the proposed PLO a large interest 


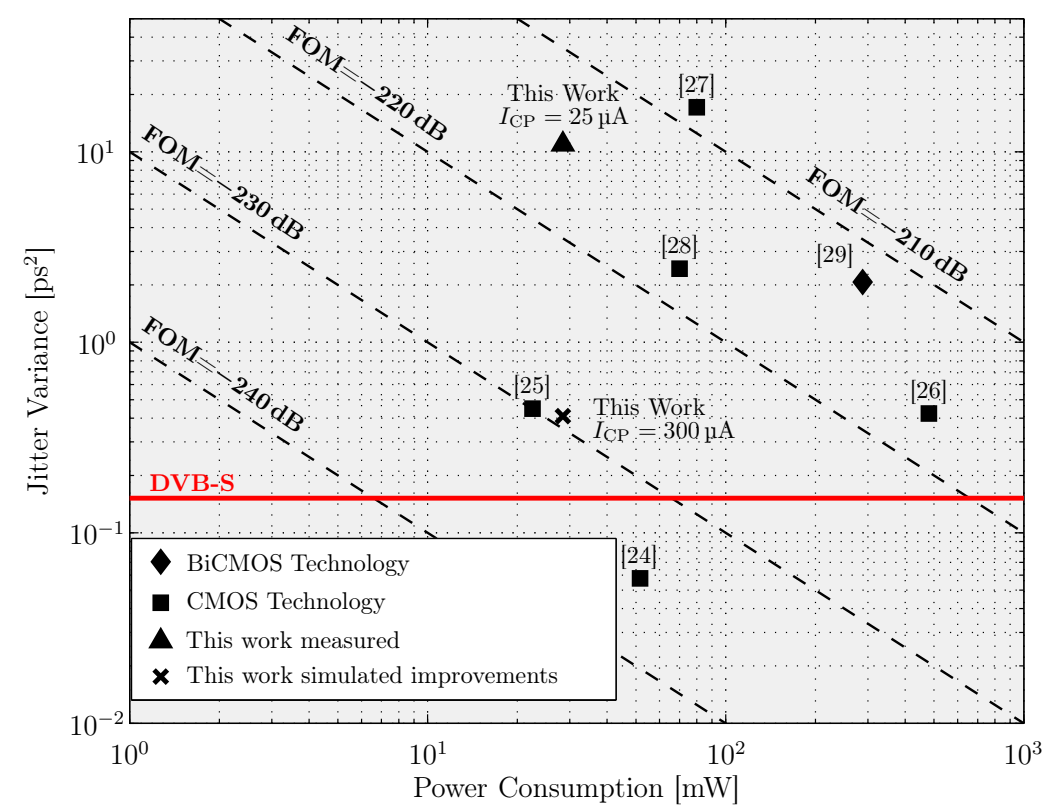

Figure 12: Jitter and power comparison between the PLO reported in this work and others PLL reported in the literature in term of $F o M_{P L L}$.

in the case of mobile and/or energy self-sustaining applications where the dissipated power should be kept as low as possible.

\section{Conclusions}

This paper reports on the design of a low-power integer-N PLO fabricated in a low cost $130 \mathrm{~nm}$ CMOS technology. The proposed PLO operates in the Ku-band, generating an output tone in the $14.16 \div 15.12 \mathrm{GHz}$ frequency range. The power delivered to a $50 \Omega$ load is about $-16.8 \mathrm{dBm}$ and the phase noise is $-86.3 \mathrm{dBc} / \mathrm{Hz}$ for an offset frequency of $1 \mathrm{MHz}$ and of $-122.2 \mathrm{dBc} / \mathrm{Hz}$ for an offset frequency of $10 \mathrm{MHz}$. The PLO sinks $23.7 \mathrm{~mA}$ from $1.2 \mathrm{~V}$ supply. The discussion of the experimental data points out that the charge pump is the main responsible for the measured phase noise level. Once compared in terms of jitter with other silicon-based PLOs and PLLs reported in the literature, the PLO of this work is well aligned if the charge pump current is set to a value that keeps low the silicon area for the loop filter. For higher charge pump current the PLO performs better than a large number of the other 


\begin{tabular}{|c|c|c|c|c|c|}
\hline & This Work 1 & This Work 2 & {$[24]$} & [25] & [26] \\
\hline Process & CMOS $130 \mathrm{~nm}$ & CMOS $130 \mathrm{~nm}$ & CMOS $130 \mathrm{~nm}$ & CMOS $130 \mathrm{~nm}$ & CMOS $130 \mathrm{~nm}$ \\
\hline Frequency [GHz] & 15 & 15 & 38 & 20.05 & 19.2 \\
\hline Freq. Range $[\mathrm{GHz}]$ & $14.2 \div 15.1$ & $14.2 \div 15.1$ & $37 \div 38.5$ & $20.05 \div 21$ & $17.6 \div 19.4$ \\
\hline Supply [V] & 1.2 & 1.2 & 1.2 & 1.5 & $1.3 \div 1.5$ \\
\hline Power $[\mathrm{mW}]$ & $28.43^{*}$ & $28.43^{*}$ & $51.6^{*}$ & 22.5 & 480.4 \\
\hline Chip Area $\left[\mathrm{mm}^{2}\right]$ & $0.45 \times 0.9^{*}$ & $0.45 \times 1.3^{*}$ & $1.5 \times 1.1$ & $0.6 \times 1$ & 1.7 \\
\hline $\begin{array}{l}\text { Phase Noise } \\
{[\mathrm{dBc} / \mathrm{Hz}]}\end{array}$ & $\begin{array}{l}-68.9 @ 100 \mathrm{kHz} \\
-86.28 @ 1 \mathrm{MHz} \\
-122.3 @ 10 \mathrm{MHz} \\
\end{array}$ & $\begin{array}{l}-84.3 @ 100 \mathrm{kHz} \\
-97.17 @ 1 \mathrm{MHz} \\
-129.7 @ 10 \mathrm{MHz} \\
\end{array}$ & $\begin{array}{l}-85 @ 100 \mathrm{kHz} \\
-97.5 @ 1 \mathrm{MHz}\end{array}$ & $\begin{array}{c}-77 @ 100 \mathrm{kHz} \\
-98.5 @ 1 \mathrm{MHz} \\
-116.1 @ 10 \mathrm{MHz} \\
\end{array}$ & $\begin{array}{c}-84 @ 100 \mathrm{kHz} \\
-101.2 @ 1 \mathrm{MHz} \\
-113.5 @ 10 \mathrm{MHz} \\
\end{array}$ \\
\hline Ref. Frequency & $58.6 \mathrm{MHz}$ & $58.6 \mathrm{MHz}$ & $1.1875 \mathrm{GHz}$ & $78 \mathrm{MHz}$ & $600 \mathrm{MHz}$ \\
\hline Bandwidth $[\mathrm{Hz}]$ & $500 \mathrm{k}$ & $500 \mathrm{k}$ & $15.625 \mathrm{M}$ & $400 \mathrm{k}$ & $\approx 4 \mathrm{M}$ \\
\hline $\begin{array}{c}\text { Filter/ } \\
\text { Division_Ratio }\end{array}$ & Integrated/Fixed & Integrated/Fixed & Integrated/Fixed & Integrated/Progr & Integrated/Fixed \\
\hline $\mathrm{I}_{\mathrm{CP}}[\mu \mathrm{A}]$ & 25 & 300 & 250 & 70 & - \\
\hline $\begin{array}{c}\text { Reported jitter rms } \\
{[\mathrm{ps}]}\end{array}$ & - & - & 0.24 & - & 0.65 \\
\hline $\begin{array}{c}\text { Calculated jitter rms } \\
\text { [ps] } \\
\text { (Integration Range) }\end{array}$ & $\begin{array}{c}3.31 \\
(10 \mathrm{kHz} \div 40 \mathrm{MHz})\end{array}$ & $\begin{array}{c}0.64^{* *} \\
(10 \mathrm{kHz} \div 40 \mathrm{MHz})\end{array}$ & $\begin{array}{c}0.36 \\
(10 \mathrm{kHz} \div 40 \mathrm{MHz})\end{array}$ & $\begin{array}{c}0.67 \\
(50 \mathrm{kHz} \div 40 \mathrm{MHz})\end{array}$ & $\begin{array}{c}0.78 \\
(10 \mathrm{kHz} \div 40 \mathrm{MHz})\end{array}$ \\
\hline \multirow[t]{2}{*}{ FoM $[22]$} & -215.1 & $-229.3^{* *}$ & -235.3 & -230 & -216.9 \\
\hline & [27] & [28] & {$[29]$} & {$[30]$} & \\
\hline Process & CMOS $65 \mathrm{~nm}$ & CMOS $180 \mathrm{~nm}$ & $\begin{array}{c}\text { SiGe:C } \\
\text { BiCMOS } \\
0.25 \mu \mathrm{m} \\
\end{array}$ & $\begin{array}{l}\text { SiGe:C } \\
\text { BiCMOS } \\
0.13 \mu \mathrm{m} \\
\end{array}$ & \\
\hline Frequency [GHz] & 20.88 & 15 & 15.75 & 20.76 & \\
\hline Freq. Range $[\mathrm{GHz}]$ & $19.44 \div 21.6$ & $13.9 \div 15.6$ & $14.25 \div 15.75$ & $20.51 \div 21.27$ & \\
\hline Supply $[\mathrm{V}]$ & $1.2 \div 1.8$ & 1.8 & $2.5 \div 1.5$ & 1.5 & \\
\hline Power $[\mathrm{mW}]$ & 80 & 70 & 288 & 40 & \\
\hline Chip Area $\left[\mathrm{mm}^{2}\right]$ & $1.6 \times 1.9$ & 1 & $0.7 \times 0.8$ & $0.84 \times 0.57$ & \\
\hline $\begin{array}{l}\text { Phase Noise } \\
{[\mathrm{dBc} / \mathrm{Hz}]}\end{array}$ & $\begin{array}{l}-65 @ 100 \mathrm{kHz} \\
-100 @ 1 \mathrm{MHz} \\
-126 @ 10 \mathrm{MHz}\end{array}$ & $\begin{array}{l}-73 @ 100 \mathrm{kHz} \\
-103.8 @ 1 \mathrm{MHz}\end{array}$ & $\begin{array}{l}-68.66 @ 100 \mathrm{kHz} \\
-97.17 @ 1 \mathrm{MHz}\end{array}$ & $\begin{array}{l}-68.66 @ 100 \mathrm{kHz} \\
-97.17 @ 1 \mathrm{MHz}\end{array}$ & \\
\hline Ref. Frequency & $36 \mathrm{MHz}$ & $71 \mathrm{MHz}$ & $250 \mathrm{MHz}$ & $81.1 \mathrm{MHz}$ & \\
\hline Bandwidth [Hz] & $\approx 70 \mathrm{k}$ & $200 \mathrm{k}$ & $\approx 3 \mathrm{MHz}$ & - & \\
\hline $\begin{array}{c}\text { Filter/ } \\
\text { Division Ratio }\end{array}$ & External/Progr & Integrated/Progr & Integrated/Progr & Integrated/Fixed & \\
\hline $\mathrm{I}_{\mathrm{CP}}[\mu \mathrm{A}]$ & - & 600 & - & 100 & \\
\hline $\begin{array}{c}\text { Reported jitter rms } \\
{[\mathrm{ps}]}\end{array}$ & - & - & - & - & \\
\hline $\begin{array}{l}\text { Calculated jitter rms } \\
\text { [ps] } \\
\text { (Integration Range) }\end{array}$ & $\begin{array}{c}4.14 \\
(10 \mathrm{kHz} \div 40 \mathrm{MHz})\end{array}$ & $\begin{array}{c}1.56 \\
(10 \mathrm{kHz} \div 40 \mathrm{MHz})\end{array}$ & $\begin{array}{c}1.44 \\
(10 \mathrm{kHz} \div 40 \mathrm{MHz})\end{array}$ & - & \\
\hline FoM $[22]$ & -208.6 & -217.7 & -212.3 & - & \\
\hline
\end{tabular}

Table 1: Performances comparison summary with other silicon-based PLL.

PLOs and PLLs, even if this high level of performances goes with an increase of the loop filter silicon area. These results demonstrate that a low-cost $130 \mathrm{~nm}$ CMOS technology is very promising for DVBS, where only discrete components or SiGe technologies have been employed until now. In addition, the reported prototype opens also the way to the fabrication of the front-end of a $\mathrm{Ku}$-band microwave radiometer. For this application, the interest of the proposed PLO comes from the large reduction of the antenna size with respect to X-band radiometer, because of the higher operation frequency, and from its low dissipated power. The proposed PLO is therefore interesting for use in a smart sensor network for the detection of wild fire in outdoor environment. In this case, the low DC consumption is very welcome to supply 
the electronics with a small dimension energy harvester. In summary, the PLO reported in the present work demonstrates that DVB-S compliant phase noise performance can come together with a low power dissipation in a PLL designed in a bulk low cost 130nm CMOS technology.

\section{References}

[1] G. Girlando, S. Smerzi, T. Copani, G. Palmisano, A monolithic 12-GHz heterodyne receiver for DVB-S applications in silicon bipolar technology, IEEE Transactions on Microwave Theory and Techniques 53 (2005) 952 -959 .

[2] P. Philippe, L. Praamsma, R. Breunisse, E. van der Heijden, F. Meng, S. Bardy, F. Moreau, S. Wane, E. Thomas, A low power 9.75/10.6GHz down-converter IC in SiGe:C BiCMOS for ku-band satellite LNBs, in: 2011 IEEE Bipolar/BiCMOS Circuits and Technology Meeting (BCTM), pp. $211-214$.

[3] Z. Deng, J. Chen, J. Tsai, A. Niknejad, A CMOS ku-band singleconversion low-noise block front-end for satellite receivers, in: IEEE Radio Frequency Integrated Circuits Symposium, 2009. RFIC 2009, pp. $135-138$.

[4] K. Miyashita, A ku-band down-converter with perfect differential PLL in $0.18 \mu \mathrm{m}$ CMOS, in: Proceedings of 2010 IEEE International Symposium on Circuits and Systems (ISCAS), pp. $4289-4292$.

[5] A companion guide to DVB-S2, Tandberg Television (UK), 2004.

[6] M. Borgarino, A. Polemi, A. Mazzanti, Low-cost integrated microwave radiometer front-end for industrial applications, IEEE Transactions on Microwave Theory and Techniques 57 (2009) 3011 -3018.

[7] C. Patterson, T. Thrivikraman, A. Yepes, S. Begley, S. Bhattacharya, J. Cressler, J. Papapolymerou, A lightweight organic x-band active receiving phased array with integrated SiGe amplifiers and phase shifters, IEEE Transactions on Antennas and Propagation 59 (2011) 100 -109.

[8] L. Vincetti, A. Polemi, M. Zoboli, Microstrip array antenna for firedetection applications, in: 2007 IEEE Antennas and Propagation Society International Symposium, pp. $2128-2131$. 
[9] M. Ferri, D. Pinna, E. Dallago, P. Malcovati, Integrated micro-solar cell structures for harvesting supplied microsystems in 0.35- $\mu \mathrm{m}$ CMOS technology, in: 2009 IEEE Sensors, pp. 542-545.

[10] D. Titz, F. B. Abdeljelil, S. Jan, F. Ferrero, C. Luxey, P. Brachat, G. Jacquemod, Design and Characterization of CMOS On-Chip Antennas for $60 \mathrm{GHz}$ Communications, Radioengineering 21 (2012) 324 332 .

[11] http://cmp.imag.fr/products/ic/?p=sthcmos9, Last Update 26th 2010.

[12] O. Mazouffre, Y. Deval, B. Goumballa, D. Belot, J. Begueret, $23 \mathrm{GHz}$ fully integrated CMOS synthesizer, in: 9th International Conference on Solid-State and Integrated-Circuit Technology, 2008. ICSICT 2008, pp. $1581-1584$.

[13] J. Colomer, A. Saiz-Vela, P. Miribel-Catala, M. Viladoms, M. PuigVidal, J. Samitier, Efficient power conditioning circuit for self-powered microsystems (SPMS) based on a low-voltage low-power $0.13 \mu \mathrm{m}$ technology, in: 2006 IEEE International Symposium on Industrial Electronics, volume 2, pp. 897-902.

[14] P. Lucchi, D. Dermit, G. Jacquemod, J. B. Begueret, M. Borgarino, 15 $\mathrm{GHz}$ quadrature voltage controlled oscillator in $130 \mathrm{~nm}$ CMOS technology, International Journal of Microwave and Wireless Technologies 3 (2011) 627-631.

[15] P. Heydari, R. Mohanavelu, Design of ultrahigh-speed low-voltage CMOS CML buffers and latches, IEEE Transactions on Very Large Scale Integration (VLSI) Systems 12 (2004) 1081 -1093.

[16] V. Karam, J. W. M. Rogers, A $5.8 \mathrm{~mW}$ fully integrated $1.5 \mathrm{GHz}$ synthesizer in 0.13- $\mu \mathrm{m}$ CMOS, in: 2007 Topical Meeting on Silicon Monolithic Integrated Circuits in RF Systems, pp. 127-130.

[17] C. Cao, K. O, A power efficient 26-GHz 32:1 static frequency divider in 130-nm bulk CMOS, IEEE Microwave and Wireless Components Letters 15 (2005) $721-723$.

[18] K. Sengupta, H. Hashemi, Maximum frequency of operation of CMOS static frequency dividers: Theory and design techniques, in: 13th IEEE 
International Conference on Electronics, Circuits and Systems, 2006. ICECS '06, pp. 584-587.

[19] N. Ismail, M. Othman, CMOS phase frequency detector for high speed applications, in: 2009 International Conference on Microelectronics (ICM), pp. $201-204$.

[20] B. Razavi, Design of Analog CMOS Integrated Circuits, McGraw-Hill Education (India) Pvt Limited, 2002.

[21] D. Dermit, CMOS Digital Phase Locked Loops and Power Amplifiers, $\mathrm{PhD}$ thesis in information and communication technologies, XXIII cycle, University of Modena and Reggio Emilia, Italy, 2011.

[22] X. Gao, E. Klumperink, P. Geraedts, B. Nauta, Jitter analysis and a benchmarking figure-of-merit for phase-locked loops, IEEE Transactions on Circuits and Systems II: Express Briefs 56 (2009) $117-121$.

[23] J. Rogers, C. Plett, F. Dai, Integrated Circuit Design for High-Speed Frequency Synthesis, Artech House, 2006.

[24] C. L. Lan-Chou Cho, A 1.2-v 37-38.5-GHz eight-phase clock generator in 0.13- $\mathrm{m}$ CMOS technology, Solid-State Circuits, IEEE Journal of (2007) $1261-1270$.

[25] Y. Ding, K. Kenneth, A 21-GHz 8-modulus prescaler and a 20-GHz phase-locked loop fabricated in 130-nm CMOS, Solid-State Circuits, IEEE Journal of 42 (2007) $1240-1249$.

[26] J. Kim, J.-K. Kim, B.-J. Lee, N. Kim, D.-K. Jeong, W. Kim, A 20-GHz phase-locked loop for $40-\mathrm{gb} / \mathrm{s}$ serializing transmitter in $0.13 \mu \mathrm{m}$ CMOS, IEEE Journal of Solid-State Circuits 41 (2006) 899 - 908.

[27] O. Richard, A. Siligaris, F. Badets, C. Dehos, C. Dufis, P. Busson, P. Vincent, D. Belot, P. Urard, A 17.5-to-20.94GHz and 35-to-41.88GHz PLL in 65nm CMOS for wireless HD applications, in: Solid-State Circuits Conference Digest of Technical Papers (ISSCC), 2010 IEEE International, pp. $252-253$. 
[28] Y.-H. Peng, L.-H. Lu, A 16-GHz triple-modulus phase-switching prescaler and its application to a $15-\mathrm{GHz}$ frequency synthesizer in 0.18 $\mu \mathrm{m}$ CMOS, IEEE Transactions on Microwave Theory and Techniques 55 (2007) $44-51$.

[29] J.-Y. Lee, S.-H. Lee, H. Kim, H.-K. Yu, A 15-GHz 7-channel SiGe:C PLL for $60-\mathrm{GHz}$ WPAN application, in: 2007 IEEE Radio Frequency Integrated Circuits (RFIC) Symposium, pp. $537-540$.

[30] J. He, J. Li, D. Hou, Y.-Z. Xiong, D. Yan, M. Arasu, M. Je, A 20$\mathrm{GHz}$ VCO for PLL synthesizer in 0.13- $\mu \mathrm{m}$ BiCMOS, in: 2012 IEEE International Symposium on Radio-Frequency Integration Technology (RFIT), pp. $231-233$. 